\title{
Delivering Sign Language in a Live Planetarium Show Using Head-Mounted Displays and Infrared Light
}

\author{
Michael D. Jones \\ Brigham Young U. \\ Provo, Utah, USA \\ jones@cs.byu.edu
}

\author{
M. Jeannette Lawler \\ Brigham Young U. \\ Provo, Utah, USA \\ jeannette_lawler@byu.edu
}

\begin{abstract}
Sign language narration is difficult to view at live planetarium shows because the room is dark and the signer is not located near images projected onto the planetarium dome. We have designed and implemented a system using head-mounted displays (HMDs) and infrared light to support viewing real-time sign language narration of live planetarium shows. Results from a series of 3 studies involving 29 students who are deaf or hard-of-hearing suggest that viewing properly configured video of sign language narration in an HMD may increase learning in this setting. Participants expressed no single preference regarding signer position in the video feed but did indicate that the relative brightness of the HMD must be tuned to match the apparent brightness of images broadcast on the planetarium dome. We also identified other issues related to HMD fit and the appearance of the signer in the video.
\end{abstract}

\section{Author Keywords}

Sign language; head mounted display; planetarium.

\section{ACM Classification Keywords}

Human-centered computing Accessibility systems and tools

\section{INTRODUCTION}

In this paper, we describe a system for delivering American Sign Language (ASL) in a live planetarium show. A live show involves a narrator located in the planetarium down who provides real-time narration during the show. Planetariums are challenging environments because the room is dark and visual targets are distributed on the planetarium dome away from a signer positioned on the planetarium floor.

Live signers are particularly common in "star shows" in which viewers look at projected stars that mimic the night sky while the narrator describes elements of that scene. Not

Permission to make digital or hard copies of all or part of this work for personal or classroom use is granted without fee provided that copies are not made or distributed for profit or commercial advantage and that copies bear this notice and the full citation on the first page. Copyrights for components of this work owned by others than the author(s) must be honored. Abstracting with credit is permitted. To copy otherwise, or republish, to post on servers or to redistribute to lists, requires prior specific permission and/or a fee. Request permissions from Permissions@acm.org.

ASSETS '19, October 28-30, 2019, Pittsburgh, PA, USA

(C) 2019 Copyright is held by the owner/author(s). Publication rights licensed to ACM.

ACM 978-1-4503-6676-2/19/10 ..\$15.00

https://doi.org/10.1145/3308561.3353809 all star shows are live; and not all live shows are star shows. Star shows can be important because they connect viewers to the stars as they seem them in a dark sky outdoors. Star shows can also be a critical part of descriptive astronomy education. We aim to make live star shows more accessible to people who are deaf or hard-of-hearing (DHH).

A variety of accommodations have been developed to deliver ASL in planetariums. Placing a signer on the floor creates new problems as it requires audience members to alternate between looking up at the planetarium dome and looking at the signer. Video of the signer could be projected onto the planetarium dome directly but this creates significant light pollution that obscures dim objects and requires all viewers to look in the same place. Even a little light pollution can wash out dim stars on the planetarium dome. Also, written captions may work well for viewers who are fluent readers, and have astronomy vocabulary in the written language---but do not replace ASL for many viewers.

We implemented a system for capturing, distributing and viewing ASL during live planetarium shows using head mounted displays (HMDs) and infrared (IR) light. The system captures the signer on camera and broadcasts the realtime video to HMDs worn by viewers. The HMDs are modified with red filters to reduce the relative brightness of the signer video as seen by the wearer. The HMD allows the viewer to see the ASL narration wherever they look and IR light is not visible to the naked eye.

We conducted a series of 3 studies with different audiences and a total of 29 students age 12-18 who are deaf or hard of hearing $(\mathrm{DHH})$ in the United States. All study participants attend schools where ASL is the primary language.

Some participants appreciated seeing ASL narration wherever they looked. Several participants identified problems with display brightness and with the appearance of the signer in the HMD. Results of the final study suggest that a properly configured video feed viewed in an HMD may improve learning in live planetarium shows compared to viewing a signer seated on the planetarium floor.

\section{RELATED WORK}

Improving Planetarium Access for People who are DHH Early work involving planetarium accessibility involved displaying captions in the planetarium [1,2,3,11]. These include displaying captions on the horizon of the dome (located at the bottom of the dome in most planetariums) 
$[2,11]$, at the viewer's seat [3] or next to visuals on the planetarium dome [1]. In [11], Shea reported that audience members who were DHH would have preferred captions placed next to visuals on the dome. Our work uses an HMD to position ASL video narration next to whatever the viewer is looking at on the dome because the HMD moves with the viewer's head.

Jones et al. investigated the use of HMDs to view ASL in pre-recorded planetarium shows [8]. They found that HMDs are a viable method for viewing ASL but this work relied on occlusive and see-through monocular displays that were available at the time. Hintz et al. measured recall of new signs taught as part of a prerecorded planetarium show also using monocular occlusive and see-through displays [4]. These two results suggest that monocular HMDs may be useable in prerecorded planetarium shows. In this paper, we consider live planetarium shows and binocular displays.

\section{HMDs for People who are DHH}

Another body of work uses HMDs to augment conversational experiences for people who are $\mathrm{DHH}$ by adding directional cues [6] or captions $[5,7,9]$. Much of this work takes place indoors $[5,6,9]$ but one study takes place outdoors [7].

Indoor conversations are simpler environments for delivering captions because participants are seated at or near eye level — usually around a table — and because lighting is controlled. Outdoor conversations present a more difficult environment due to variations in lighting. In [7], several participants mentioned difficulties associated with brightness of sunlight outdoors.

\section{THE SYSTEM}

Our objective is to provide comprehensible video of live ASL narration of a planetarium show viewed in an HMD. We decomposed this objective into four requirements and implemented a system guided by those requirements.

\section{Requirements}

Design requirements are grounded in the objective, results from prior work and the experiences of members of the system design and implementation team. The team consisted of 3 people who are hearing and 3 people who are DHH.

\section{Video Quality}

Video quality is measured in terms of frame resolution in pixels, frame rate and encoded bitstream size in mb per second. From a usability perspective, video quality should be high enough to provide ASL that the viewer can understand but is constrained by the display resolution of the HMD and bandwidth of the transmission network. We used Weaver et al.'s study of video resolution and sign language comprehension on smartphone screens as a guide [12]. Weaver et al. found that novice ASL viewers could comprehend ASL on a smartphone screen at resolutions of $640 \times 480,320 \times 240$, or even 160x120. Fluent ASL viewers may have different video resolution needs.

\section{Live Signer}

The live signer could be an interpreter or the narration could be provided in ASL directly. We chose to use a live signer because some planetarium shows, such as star shows, use a live rather than pre-recorded narration and some planetariums lack facilities for prerecorded narration.

\section{Signer Physical Location}

The signer viewed in the HMD could be physically located in a chair on the planetarium floor, outside the planetarium or even at a remote location in a different building. After discussing possibilities with two ASL interpreters that have experience signing for planetarium shows, we chose to position the signer in the planetarium dome. This gives the signer as much context as possible during the show.

The signer viewed live by participants during the show was placed in a chair on the planetarium show directly in front of participants. The signer was illuminated with a red light. This position and lighting is common when a signer narrates or translates a planetarium show.

\section{Head-worn Displays}

We chose an HMD system because it allows each viewer to see the ASL wherever the viewer looks in the planetarium. A see-through, rather than an occlusive, display was chosen to avoid partially blocking the viewer's vision with an opaque display.

\section{Architecture and Implementation}

Guided by these requirements, we designed and implemented a system consisting of three components: camera and lighting, transmission, and displays. The camera and lighting produce a video stream which is sent by the transmitter to the display. The viewer watches the ASL in the display.

\section{Camera and Lighting}

The signer is seated in front of a black backdrop which is large enough to extend beyond the signer's box---or space in which they perform signs. The signer is lit by two infrared (IR) lights with one light dimmer than the other to provide even illumination and some contrast. A camera is positioned in front of the signer with position and focal length set to capture the signer's entire box. Because people cannot see IR light, this system provided a viewable video feed without visible light pollution.

Our implementation of the camera and lighting involved two IR floodlights which emit light at $850 \mathrm{~nm}$ and were designed for home security use. The camera was a Canon XA-10 which captures video at a resolution of $1080 \mathrm{p}$ or less and has a removable IR cut filter. Digital camera sensors detect IR light but a cut filter is added to block IR light. The XA-10 allows that filter to be easily removed from the light path. We used gaffe tape to cover all LED status indicators on all equipment to reduce light pollution.

The background fabric was a black velvet manufactured using dye which absorbs IR light---some black fabrics reflect IR light much like white fabric reflects light in the visible 
spectrum. The black fabric produces a black background which looks transparent when viewed through an HMD.

\section{Transmission}

Video from the camera passes to a video transmitter which encodes and sends the video. The transmitter controls the video quality and transmission parameters. We used Teradek Cube-255 encoders to encode and transmit video. Video was encoded at 480x360 pixels, 30 frames per second and limited to $3 \mathrm{Mbps}$. Video was wirelessly broadcast using the real-time streaming protocol (RTSP) over TCP/IP. Video resolution includes 9 times as many pixels as the lower bound on video quality for novice users reported in [12]. Video quality balanced trade-offs between the viewing experience and network transmission limits.

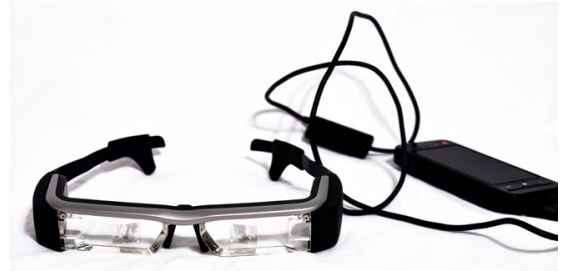

Figure 1 The Epson Moverio BT-200 HMDs used in all 3 studies. This HMD is a binocular see-through display with video projected into the glasses near the nose piece.

\section{Displays}

Video transmissions are received and displayed by HMDs given to each viewer. We used binocular see-through Epson Moverio BT-200 displays, shown in Figure 1. Displays were set to decode video without audio using a $300 \mathrm{~ms}$ buffer to minimize latency. We chose a smaller buffer with lower latency so that viewers would see ASL video in the display closer in time to when the ASL was generated by the signer. Our system achieved a latency of 2-5 seconds with occasional periods of up to 10 seconds latency.

Displays were set to display video at the lowest brightness setting. As described below, in the first study we determined that this was not dim enough for viewing ASL at all planetarium shows despite being dim enough in the planetarium used in the pilot study. Before the second and third studies, we designed red filters for the HMDs to reduce the difference between the brightness of the HMD display and the stars on the planetarium dome (see Figure 2). We added filters by removing a dust cover in each arm of the glasses. We cut a small red filter to fit in the space behind each dust cap. We 3D printed new dust caps the same size and shape as the original dust caps but with a small slit to hold the filter in place.

\section{FORMATIVE STUDIES}

\section{Methods}

After carrying out a pilot study, we conducted two formative studies of the system. In the pilot study, three members of the research team who are DHH used the system in a planetarium show with live ASL narration provided by a signer seated in the planetarium and took the post-test. The pilot study found no issues with the system itself but we did revise the study protocol to use common names for constellations rather than Greek or Latin names. We switched to common names because pilot study participants felt that using the Greek or Latin names (such as "Ursa Major" or "Taurus") turned the study into an investigation of finger spelling unfamiliar words rather than an investigation of ASL in a planetarium.

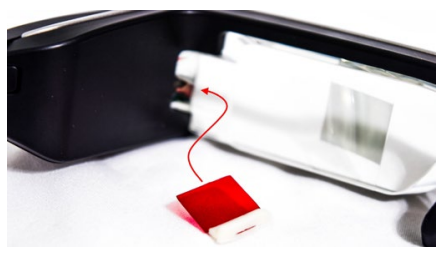

Figure 2 Inserting a red filter (as shown by the red arrow) into the projected light path reduced the brightness of the video seen in the HMD. This reduced the difference between the brightness of the video and the stars projected onto the planetarium dome in studies 2 and 3.

The purpose of the two studies was to understand the experience of viewing ASL in a HMD at a live planetarium show. The studies were serialized and iterative with results from first evaluation used to change the next evaluation. A total of 22 people participated with 7 in the first and 15 in the second studies. Studies were approved in advance by our Institutional Review Board (IRB). Parents or guardians of participants gave written consent and participants gave assent. We did not compensate participants directly but we paid for travel, interpretation and admission costs associated with the planetarium show.

For each study, we recruited students aged 12-18 from schools for the deaf in United States to view a planetarium show at a planetarium near their school. Participants in the first study came from Brazos River Day School Program for the Deaf in Texas and the American School for the Deaf in Connecticut for the second study. Studies were conducted at the Noble Planetarium in Fort Worth, Texas and the Travelers Science Dome Planetarium in West Hartford, Connecticut respectively. Both planetariums are within driving distance of students' schools. All participants communicated primarily in ASL and received instruction at school in ASL. English, Spanish or ASL was spoken in all participant homes.

Each participant in each show watched half of the show with an interpreter seated on the planetarium floor and half of the show with an interpreter displayed in an HMD. Half of the participants used the HMDs first and the other half used the HMDs second. All participants always viewed ASL generated by the same interpreter. Participants not using the HMD viewed the signer directly and were seated so that the camera did not obstruct their view. Participants viewing ASL in the HMD were seated with the interpreter out of view. 


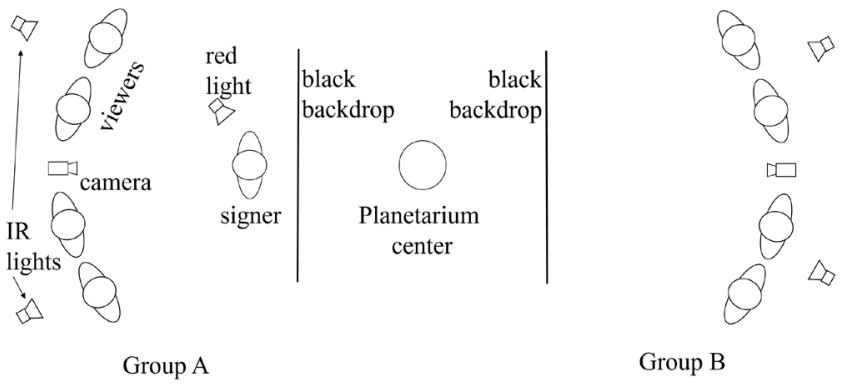

Figure 3. Setup for each of the studies. Members of groups A and $B$ both see half of the planetarium show by watching the signer seated on the floor-in front of Group $A$ in this sketchor in video broadcast to an HMD. Halfway through the show, the signer moves from Group A to Group B and the HMDs are moved from Group B to Group A.

Participants sat in the same seats during both halves of the show. We placed each group of participants on different sides of the planetarium and installed two sets of IR lights, cameras and backgrounds as shown in Figure 3. The camera is located between participants and the lights are mounted behind and above participants. A black backdrop is placed behind the signer's seats. In Figure 3, the signer is seated in front of Group A who view ASL by watching the signer in red light. Group B views the signer through a video feed broadcast to their HMDs. Halfway through the show we moved the interpreter from Group A to Group B and the HMDs switch from Group B to Group A.

The show consisted of a lecture on 18 constellation names in which the narrator used a laser pointer to highlight each constellation while describing an English name for that constellation and telling why the constellation was given that name. We selected constellations that appear to the left or right of participants to provide similar access to constellations for both groups of participants.

After the planetarium show, we asked participants to complete an examination in which we pointed out each of the 18 constellations and asked participants to recall the name of the constellation. Participants were told that there would be a test before the show. In the first evaluation, we observed participant behavior and talked with participants after the show through an interpreter.

In the second evaluation, we also gave participants a feedback form and asked for their written feedback in English. The feedback form included two open ended questions, a picture of glasses to draw upon and 3 Likert items. The open-ended questions asked what participants did or did not like about the experience of viewing ASL in an HMD. Participants were asked to draw their ideal signer placement in the picture of a pair of glasses. The Likert items measured preferences related to the brightness of the image in the glasses; and the difficulty of paying attention to ASL in the glasses or with an interpreter seated on the floor. Likert items each had 5 responses ranging from negative to positive with labels such as "very difficult" to "very easy."
Open-ended responses were reviewed by two members of the study team using thematic analysis. Some participants may not have responded because the form was in English.

\begin{tabular}{|c|c|c|c|c|}
\hline & $\boldsymbol{n}$ & $\begin{array}{c}\text { Head-mounted } \\
\text { display }\end{array}$ & $\begin{array}{c}\text { Signer } \\
\text { on floor }\end{array}$ & Ratio \\
\hline Study 1 & 7 & 20 & 37 & 0.54 \\
\hline Study 2 & 15 & 31 & 45 & 0.69 \\
\hline Study 3 & 7 & 14 & 8 & 1.75 \\
\hline
\end{tabular}

Table 1. Number of constellation names recalled by all participants in each study when viewing ASL in head-mounted displays or a signer seated on the floor. The last column gives the ratio of names recalled for each viewing scenario.

\section{Results}

\section{Relative Brightness and Recall}

Based on the pilot study, we expected that the relative brightness of the signer in the HMD compared to other visual elements in the planetarium would have a significant impact on the experience. We discussed this issue with participants in the first study who said that the video image was too bright and they could not see most of the stars projected onto the planetarium dome. Perhaps due to this problem, participants recalled fewer constellation names with the HMDs than without as shown in the row for Study 1 in Table 1. The first boxplot in Figure 4 shows the distribution of differences in the number of names recalled over all participants in Stuy 1. All participants recalled fewer constellation names when using the HMD---except 1 participant who recalled the same number of names in both viewing conditions.

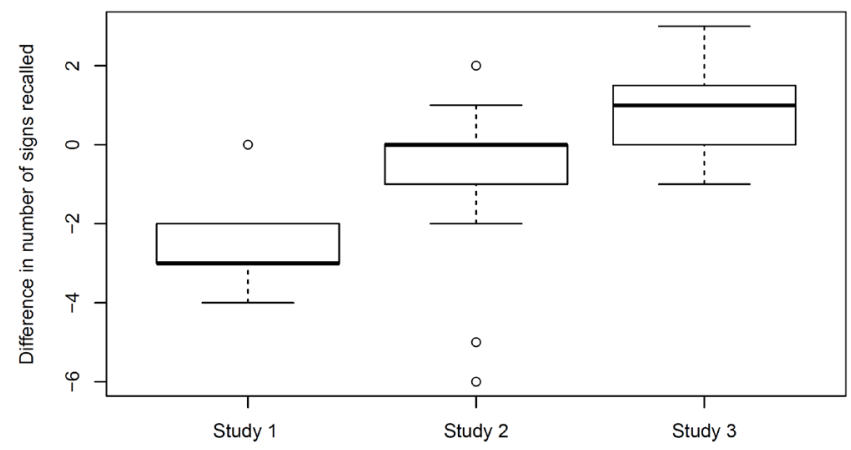

Figure 4. Distributions of differences between the number of signs recalled for each participant in each viewing condition across all 3 studies. For each participant, the difference is the number of names recalled using the HMD minus the number recalled when viewing the signer seated on the floor. Differences less than 0 indicate that those viewers recalled fewer names using the HMD.

In the second study, we added red filters into the projection path as described above and increased the brightness of the stars displayed in the planetarium.

In response to a Likert item about HMD display brightness, 5 participants in the second study said the video was too dark, 
5 said it was "just right", 3 said it was too bright and 2 gave no answer. Once again, participants recalled fewer constellation names using the HMD in this study as shown in the row for Study 2 in Table 1 and summarized in Figure 4. This reduction in recall using the HMDs may also be attributable to problems with display brightness.

\section{Likes and Dislikes}

We found three themes in responses to open-ended questions about things participants did like and did not like. On the "dislike" side, 7 participants did not like the video image because it was "blurry" or red. Participant P1 said "I don't like when they sign and [I] see only black and red." Four participants did not like the physical fit of the glasses because, for example, the glasses were "heavy" (P5).

On the "like" side, 5 participants found the video in the glasses easier to understand. One participant said it was "easier to see dome and glasses [at the] same time" (P8).

\section{Signer Video Location in HMD}

Video of the signer was positioned in the center of the display for all participants. 4 participants in the second study indicated that they preferred the signer positioned in the center of the display, 6 preferred the signed positioned outside of the center of the display (such as in the top outside corner) and 5 provided no answer.

\section{Comparative Ease of Viewing}

In response to Likert items about the ease of viewing the signer seated on the floor and the signer video in an HMD, 5 participants reported that the signer was easier to pay attention to when the signer was seated on the floor compared to viewing the signer in an HMD. 5 participants expressed the opposite preference. 3 participants reported the same ease of viewing in both conditions and 2 participants did not respond.

\section{Discussion}

In studies 1 and 2, nearly all participants recalled fewer constellation names when viewing narration through the HMD. The relative brightness of the video in the HMD may have reduced learning. If the HMD image is too bright in a star show, the viewer may not see the stars projected on the planetarium dome when the stars are dim compared to the HMD brightness. In that case, the viewer can not see the shape of the constellation and can not match that shape to the constellation name when asked. On the other hand, if the image is too dim, the viewer will not see the ASL. Providing captions in outdoors settings also encountered issues with relative brightness of the text in the HMD in sunlight [7].

Also, DHH viewers in our second study did not have a single unifying preference for signer position in the HMD. This is consistent with prior work involving captions [5,7].

\section{SUMMATIVE STUDY}

Based on studies 1 and 2, we designed a third study as a summative evaluation. In study 3 , we again modified the relative brightness of the ASL video in the HMD and the stars shown on the planetarium dome. We did this by using a different planetarium with the ability to make brighter images of stars on the planetarium dome than was possible in the other two planetariums.

The purpose of the summative evaluation was to determine if adjusting the relative brightness of the display resulted in an increase in learning when viewing ASL in an HMD compared to viewing a signer seated on the floor in a live planetarium show about constellation names. The study followed the same protocol as the first two formative studies described above but without the closing survey.

We recruited 17 participants ages 12-18 from the Horace Mann School-a public school for students who are DHH in Massachusetts. The study was conducted at the Charles Hayden Planetarium in Boston, Massachusetts. Unexpected technical issues interrupted the broadcast of video during the show which impacted 10 participants leaving us with usable data from only 7 participants. These 7 participants did not report that the video failed during the presentation.

\section{Results}

As shown in the row for Study 3 in Table 1, participants as a group correctly recalled 14 constellation names for the parts of the show they viewed in the HMD and participants correctly recalled 8 constellation names from the parts of the show viewed with the signer seated on the floor. Figure 4 shows the distribution of differences across participants.

\section{Discussion}

Nearly all participants recalled more constellation names when viewing ASL through a HMD compared to viewing a signer seated on the planetarium floor. This result suggests that viewing properly configured video of ASL in an HMD may improve the accessibility of instruction delivered in a planetarium star show. The small sample size is a limitation of our work.

\section{CONCLUSION}

Properly configured video of ASL viewed in an HMD may improve access to ASL in live planetarium shows. The relative brightness of the signer displayed in the HMD compared to the background scene is an important factor. Other factors to consider include the position of the signer within the HMD display and the HMD physical fit on the viewer's head. The maximum brightness of the planetarium projection system is a key part of balancing the brightness of the HMD video and the brightness of the projected image. Brightness of the projection systems varies widely between planetariums. The brightest system used in our studies is located the Hayden Planetarium used in study 3.

Future work might consider the role of HMDs in delivering ASL in other logistically challenging learning environments in which it is difficult to place the signer in the learner's field of view. Future work might also explore viewing ASL in an HMD in other settings---such as large lecture halls.

\section{ACKNOWLEDGMENTS}

This work supported by NSF grants IIS-1406578 and IIS1124548 and gifts from the Sorenson Impact Foundation. 


\section{REFERENCES}

[1] Lionel Daniel. 1974. Planetarium for the deaf. Planetarian. 3, 1, 7-11.

[2] Jerome DeGraff and Fred Hamil. 1972. Seeing Stars. Plaentarian. 1, 2, 54-55.

[3] Noreen Grice. 1996. Planetarium captioning system for the hearing-impaired. Planetarian. 25, 4, 8-9.

[4] Eric Hintz, Michael D. Jones, and M. Jeannette Lawler. Adoption of ASL classifiers as delivered by headmounted displays in a planetarium show. Journal of Astronomy and Earth Sciences Education. 2, 1, 1-16.

[5] Dhruv Jain, Bonnie Chinh, Leah Findlater, Raja Kushalnagar, and Jon Froehlich. 2018. Exploring Augmented Reality Approaches to Real-Time Captioning: A Preliminary Autoethnographic Study. In Proceedings of the 2018 ACM Conference Companion Publication on Designing Interactive Systems (DIS '18 Companion). ACM, New York, NY, USA, 7-11. DOI: https://doi.org/10.1145/3197391.3205404

[6] Dhruv Jain, Leah Findlater, Jamie Gilkeson, Benjamin Holland, Ramani Duraiswami, Dmitry Zotkin, Christian Vogler, and Jon E. Froehlich. 2015. HeadMounted Display Visualizations to Support Sound Awareness for the Deaf and Hard of Hearing. In Proceedings of the 33rd Annual ACM Conference on Human Factors in Computing Systems (CHI '15). ACM, New York, NY, USA, 241-250. DOI: https://doi.org/10.1145/2702123.2702393

[7] Dhruv Jain, Rachel Franz, Leah Findlater, Jackson Cannon, Raja Kushalnagar, and Jon Froehlich. 2018. Towards Accessible Conversations in a Mobile Context for People who are Deaf and Hard of Hearing. In Proceedings of the 20th International ACM SIGACCESS Conference on Computers and Accessibility (ASSETS '18). ACM, New York, NY, USA, 81-92. DOI:

https://doi.org/10.1145/3234695.3236362
[8] Michael Jones, M. Jeannette Lawler, Eric Hintz, Nathan Bench, Fred Mangrubang, and Mallory Trullender. 2014. Head mounted displays and deaf children: Facilitating Sign Language in Challenging Learning Environments. In Proceedings of the 2014 conference on Interaction design and children (IDC '14). ACM, New York, NY, USA, 317-320. DOI: https://doi.org/10.1145/2593968.2610481

[9] Yi-Hao Peng, Ming-Wei Hsi, Paul Taele, Ting-Yu Lin, Po-En Lai, Leon Hsu, Tzu-chuan Chen, Te-Yen Wu, Yu-An Chen, Hsien-Hui Tang, and Mike Y. Chen. 2018. SpeechBubbles: Enhancing Captioning Experiences for Deaf and Hard-of-Hearing People in Group Conversations. In Proceedings of the 2018 CHI Conference on Human Factors in Computing Systems (CHI '18). ACM, New York, NY, USA, Paper 293, 10 pages. DOI: https://doi.org/10.1145/3173574.3173867

[10] Halley Profita, Reem Albaghli, Leah Findlater, Paul Jaeger, and Shaun K. Kane. 2016. The AT Effect: How Disability Affects the Perceived Social Acceptability of Head-Mounted Display Use. In Proceedings of the 2016 CHI Conference on Human Factors in Computing Systems (CHI '16). ACM, New York, NY, USA, 48844895. DOI: https://doi.org/10.1145/2858036.2858130

[11] Shea. 1993. Captioning planetarium shows for the hearing impaired. Planetarian. 22, 3, 16.

[12] Kimberly A. Weaver, Thad Starner, and Harley Hamilton. 2010. An evaluation of video intelligibility for novice American Sign Language learners on a mobile device. In Proceedings of the 12th international ACM SIGACCESS conference on Computers and accessibility (ASSETS '10). ACM, New York, NY, USA, 107-114. DOI: https://doi.org/10.1145/1878803.1878824 